\title{
Dynamics of Fat Mass in DUhTP Mice Selected for Running Performance - Fat Mobilization in a Walk
}

\author{
Julia Brenmoehl ${ }^{a, b} \quad$ Daniela Ohde ${ }^{a} \quad$ Christina Walz ${ }^{a, b} \quad$ Julia Schultz ${ }^{c}$ \\ Armin Tuchscherer ${ }^{d} \quad$ Florian Rieder $^{\mathrm{e}} \quad$ Ulla Renne $^{\mathrm{b}} \quad$ Andreas Hoeflich $^{\mathrm{a}} \mathrm{b}$ \\ ${ }^{a}$ Cell Signaling Unit from the Institute for Genome Biology, Leibniz-Institute for Farm \\ Animal Biology (FBN), Dummerstorf, Germany; ${ }^{b}$ Laboratory for Mouse Genetics, Institute \\ for Genetics \& Biometry, Leibniz Institute for Farm Animal Biology (FBN), Dummerstorf,

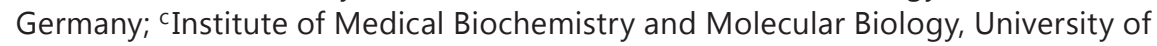 \\ Rostock, Rostock, Germany; ${ }^{d}$ Livestock Genetics and Breeding Unit, Institute for Genetics \\ \& Biometry, Leibniz-Institute for Farm Animal Biology (FBN), Dummerstorf, Germany; \\ e Department of Pathobiology, Lerner Research Institute, Cleveland Clinic Foundation, \\ Cleveland, OH, USA
}

\section{Key Words}

Adipose tissue - Subcutaneous adipose tissue - Fat loss · Mitochondrial biogenesis · Physical activity

\begin{abstract}
Objective: Reduction of body fat can be achieved by dietary programs and/or aerobic exercise training. More convenient methods to rid the body of excess fat are needed. However, it is unclear whether it is possible to more easily lose body weight at all. Methods: DUhTP mice bred through phenotype selection for high treadmill performance and unselected controls were voluntarily physically active in a running wheel over a period of 3 weeks. Phenotypical data were collected, and subcutaneous fat was analyzed for expression of mitochondria-relevant proteins. Results: Voluntary physical activity over 3 weeks exclusively in DUhTP mice severely reduced subcutaneous $(-38 \% ; p<0.05)$ and epididymal $(-32 \% ; p<0.05)$ fat. Following mild physical activity, subcutaneous fat derived from DUhTP mice showed increased levels of long chain acyl dehydrogenase (LCAD; $+230 \% ; p<0.05)$ and peroxisome proliferator-activated receptor gamma coactivator 1-alpha (PGC1- $\alpha ; p<0.01$ ). Mitochondrial transcription factor A (Tfam) expression was similar in both sedentary genotypes but physical activity in-
\end{abstract}

Julia Brenmoehl and Daniela Ohde contributed equally to the paper. 
creased Tfam levels exclusively in DUhTP $(p<0.05)$. Conclusion: Our findings indicate that the mitochondrial mass is highly active in DUhTP mice and responsive even to mild physical activity. While genetic predisposition could not prevent fat accretion in DUhTP mice, voluntary activity was sufficient to reduce excess body fat almost completely.

(c) 2015 S. Karger GmbH, Freiburg

\section{Introduction}

Surplus energy is stored in the form of lipids in white adipose tissue (WAT). Hence WAT mass can be used as a marker of positive energy balance in a given organism [1]. Under conditions of negative energy balance, e.g. during physical exercise, elevated lipolytic activity in WAT results in an increase of non-esterified ('free' or unsaturated) fatty acids in the circulation, which serve as the main energy source of the muscle [2]. With increasing energy demands, for example during high-intensity physical exercise, fat and glycogen from the muscle get recruited for energy production $[3,4]$. Utilization of energy from fat stores can be augmented by distinct resistance exercise training protocols $[5,6]$. Lipolytic activity in WAT can be induced or blocked by growth hormone, cortisol, interleukin- 6 , $\beta$-adrenergic signaling or insulin $[2,4]$. Different pharmacological approaches have been tested concerning their potential to induce fat degradation, e.g., L-carnithin, polyphenols, and others [7].

An increase of WAT mass was also observed in our mouse model, generated by long-term selection for high treadmill performance (DUhTP mice [8]). As shown previously, DUhTP mice have the same voluntary activity if running wheels (RWs) were included in their home cages [8]. Thus obesity in DUhTP mice seems not being related to altered physical activity. DUhTP mice are characterized by about fourfold increased endurance running capacity, increased hepatic lipogenesis, hyperlipidemia, and massive accumulation of body fat in external fat depots compared to unselected control mice [8]. Their fat depots contain high amounts of energy, exceeding the requirements of even ultra-long distance runs or extreme resistance training.

While control of exercise-related energy metabolism has been intensively studied in muscle, adipose tissue has received only limited attention [9]. In rat muscles, endurance training leads to increases of tricarboxylic acid cycle activity, $\beta$-oxidation, and oxidative phosphorylation [10-12]. Specific enzyme activity providing $\beta$-oxidation, such as palmityl CoA dehydrogenase and carnithine palmitoyltransferase (CPT1), increases in response to exercise [12]. In women, prolonged physical activity resulted in an increase of medium and very long chain acyl CoA (MCAD) dehydrogenase in skeletal muscles [13]. However, glycerol release from muscle is small, compared to release from subcutaneous adipose tissue during moderateand high-intensity exercise [14]. Both, physical activity and exercise can induce metabolic activity and mitochondrial biogenesis also in adipose tissues. Specifically, citrate synthase activity [15], mRNA expression of peroxisome proliferator-activated receptor gamma coactivator 1-alpha (PGC1- $\alpha$ ) as well as cytochrome $c$ oxidase and mitochondrial content have been found to be regulated by exercise in adipose tissues [16, 17]. Exercise-related induction of mitochondrial biogenesis and glucose uptake in subcutaneous fat tissues are dependent on endothelial nitric-oxide synthase (eNOS) as described previously [16].

Key regulators of lipid oxidation (MCAD, CPT1) are increased in muscle through an increase in PGC1- $\alpha[18,19]$ as shown in muscles of exercising DUhTP mice [20]. PGC1- $\alpha$ is a known inducer of mitochondrial mass and increases the amounts of mitochondrial DNA (mtDNA) $[19,21,22]$. Furthermore, PGC1- $\alpha$ coordinately induces expression of the mitochondrial transcription factor (Tfam) enhancing the replication of mtDNA and transcription of mitochondria-encoded genes [23]. In response to exercise, expression of Tfam or of mitochondria- and nucleus-encoded mitochondrial proteins was increased in muscles and adipose 
Brenmoehl et al.: Dynamics of Fat Mass in DUhTP Mice Selected for Running

Performance - Fat Mobilization in a Walk

tissue of rats, mice, and humans [15, 24-26]. We thus hypothesized that energy metabolism in WAT is altered in DUhTP mice in a manner improving muscle supply with energy, in particular fatty acids. In order to test the hypothesis of an increased energy-metabolic activity in WAT of DUhTP mice, we studied expression of lipid metabolic key enzymes and mitochondrial biogenesis in the presence and absence of RWs. By means of long-term selection in DUhTP mice, we demonstrate that it is possible to metabolize excess body fat in a walk.

\section{Material and Methods}

Animals

All in vivo experiments were performed in accordance with national and international guidelines and were approved by our internal institutional review board. In this study, we used a mouse model that has been generated by selection over 90 generations for high treadmill performance (marathon mice: DUhTP) and random selection (control mice: DUC). Both mouse lines were bred from the identical base population [8] avoiding inbreeding. In the original base population, 4 different outbred (NMRI orig., Han:NMRI, CFW, CF1) and 4 different inbred mouse lines (CBA/Bln, AB/Bln, C57BL/Bln, XVII/Bln) were included. The animals were housed in Makrolon cages Type II (EBECO, Castrop-Rauxel, Germany) under controlled environmental conditions in a semi-barrier system. Mice received fresh tap water and fixed formula food ad libitum (Altromin ${ }^{\circledR} 1314$, Altromin GmbH, Lage, Germany). Body composition was quantified by dual energy X-ray absorptiometry (Lunar PIXImus II, GE Medical Systems, Solingen, Germany).

\section{Running Wheel Performance}

Physical activity was assessed over a period of 3 weeks in 49- to 70-day-old male DUhTP mice and controls by including RWs (diameter $=33.4 \mathrm{~cm}$; Tecniplast, Hohenpeißenberg, Germany) in their home cages. A control group of mice was kept in cages without RWs. Mice were fasted overnight, killed by decapitation $(\mathrm{N}=10)$, and serum samples were collected. Tissues were weighted, snap-frozen in liquid nitrogen, and stored at $-70^{\circ} \mathrm{C}$ for subsequent analysis.

Analysis of Triglycerides and Cholesterol

Triglycerides (TG) and cholesterol were assayed in serum and liver samples by using commercial kits (triglycerides: No. LT-TR 0015, total cholesterol: No. LT-CH 0031; both Labor \& Technik Eberhard Lehmann, Berlin, Germany) as described previously [8].

Quantitative Real Time-PCR (qPCR)

Expression of PGC1- $\alpha$ mRNA transcripts in subcutaneous fat samples ( $\mathrm{N}=7$ ) was analyzed as described previously [20] and primers used are summarized in table 1. Different housekeeping genes (HKGs; Hprt1, Rplp2, and $\beta$-actin) were tested, identifying the HKGs with comparable Crossing point (Cp) values (Cp $\mathrm{Pplp}_{\text {Rp: }}$ : DUhTP mice $=24.45 \pm 1.17$; DUC mice $=25.80 \pm 1.03$ ) to normalize expression of PGC1 $-\alpha$. Mitochondrial DNA (mtDNA) was determined by comparison of gene expression levels of cytochrome B mtDNA and large ribosomal protein p0 (36B4).

\section{Immunoblotting}

Western immunoblotting was performed as described previously [20]. Equal loading of the gels and proper transfer of the proteins to the membranes were verified by Coomassie blue staining. We analyzed the expression of PGC1- $\alpha$, fatty acid synthase (FAS), long chain acyldehydrogenase (LCAD), Tfam, sirtuin 3 (SIRT3), and NADH-ubiquinone oxidoreductase alpha subunit 9 (NDUFA9) of complex I by using specific antibodies (table 2).

Mitochondrial Morphology in Adipose Tissue

Mitochondrial staining was performed on $5 \mu \mathrm{m}$ thick frozen tissue sections using MitoTracker ${ }^{\circledR}$ Deep Red (Molecular Probes ${ }^{\circledR}$; Invitrogen, Darmstadt, Germany) for $30 \mathrm{~min}$ at $37^{\circ} \mathrm{C}$. Tissues were fixed in ice-cold acetone $\left(-20^{\circ} \mathrm{C}\right)$ for $10 \mathrm{~min}$ [27]. All sections were counterstained with Dapi (Vector Laboratories, Burlingame, CA, USA), and mitochondrial morphology was observed using a confocal laser-scanning microscope Fluoview FV10i (Olympus, Hamburg, Germany). 
Brenmoehl et al.: Dynamics of Fat Mass in DUhTP Mice Selected for Running Performance - Fat Mobilization in a Walk

Table 1. Sequences of primer sets used for amplification of specific cDNA

\begin{tabular}{|c|c|c|}
\hline Name & $5^{\prime} \rightarrow 3^{\prime}$ & To detect \\
\hline $\begin{array}{l}\text { PGC1- } \alpha 1 \text {-forw } \\
\text { PGC1- } \alpha 1 \text {-rev }\end{array}$ & $\begin{array}{l}\text { ggacatgtgcagccaagactct } \\
\text { cacttcaatccacccagaaagct }\end{array}$ & PGC-1a isoform 1, inducer of browning \\
\hline $\begin{array}{l}\text { Hprt1-forw } \\
\text { Hprt1-rev }\end{array}$ & $\begin{array}{l}\text { tcctcctcagaccgctttt } \\
\text { cctggttcatcatcgctaatc }\end{array}$ & hypoxanthine guanine phosphoribosyl transferase 1 \\
\hline $\begin{array}{l}\text { Rplp2-forw } \\
\text { Rplp2-rev }\end{array}$ & $\begin{array}{l}\text { cagtctagagctcctggaaggt } \\
\text { tgtggaaaacagcaggttagc }\end{array}$ & ribosomal protein, large P2 \\
\hline $\begin{array}{l}\text { CytB-forw } \\
\text { CytB-rev }\end{array}$ & $\begin{array}{l}\text { cttcgctttccacttcatcttacc } \\
\text { ttgggttgtttgatcctgtttcg }\end{array}$ & cytochrome B \\
\hline $\begin{array}{l}\text { 36B4-forw } \\
\text { 36B4-rev }\end{array}$ & $\begin{array}{l}\text { aggatatgggattcggtctcttc } \\
\text { tcatcctcctgcttaagtgaacaaact }\end{array}$ & large ribosomal protein $\mathrm{p} 0$ \\
\hline $\begin{array}{l}\text { beta-actin-forw } \\
\text { beta-actin-rev }\end{array}$ & $\begin{array}{l}\text { tgacaggatgcagaaggaga } \\
\text { cgctcaggaggagcaatg }\end{array}$ & actin, beta, cytoplasmatic \\
\hline
\end{tabular}

Table 2. Primary antibodies used for western immunoblotting

\begin{tabular}{lll}
\hline Name & Catalogue number & Company \\
\hline PGC1- $\alpha$ & sc-13067 & Santa Cruz Biotechnology \\
FAS & sc-20140 & Santa Cruz Biotechnology \\
LCAD & sc-82466 & Santa Cruz Biotechnology \\
Tfam & sc-23588 & Santa Cruz Biotechnology \\
ND-1 & sc-20493 & Santa Cruz Biotechnology \\
NDUFA9 & sc-58392 & Santa Cruz Biotechnology \\
SIRT3 & sc-99143 & Santa Cruz Biotechnology \\
\hline
\end{tabular}

\section{Statistical Analysis}

The data analysis was performed using SAS software (Version 9.4 for Windows, SAS Institute Inc., Cary, NC, USA). Descriptive statistics and tests for normality were calculated with the UNIVARIATE procedure of Base SAS software (Base SAS ${ }^{\circledR}$ 9.4 Procedures Guide, 2nd ed; SAS Institute Inc.). Data considered as approximately normal were analyzed by ANOVA using the GLIMMIX procedure of SAS/STAT software (SAS/STAT ${ }^{\circledR}$ 13.1 User's Guide; SAS Institute Inc.). The ANOVA model for the 70-day data contained the fixed factors line (levels: DUC, DUhTP), group (levels: control, RW), and the interaction line $\times$ group. The model for the variables rel_subcutaneous_fat, rel_epididymal_fat, rel_perinephric_fat, and rel_brown_fat included the fixed effects line (levels: DUC, DUhTP), group (levels: 49 days, 70 days, 70 days + RWs), and the interaction line $\times$ group. Homogeneity of covariance parameters across groups was tested for all ANOVA models. In addition, least-squares means (LSM) and their standard errors (SE) were computed for each fixed effect in the models, and all pairwise differences of LS means were tested by the Tukey-Kramer procedure. Effects and differences were considered significant if $\mathrm{p}<0.05$.

\section{Results}

\section{Physical Changes in DUhTP Mice in Response to Moderate Physical Activity}

DUhTP mice accomplished 3,935 \pm 1,719 revolutions per day (r/day) at an age of 70 days, which was comparable to control mice $(3,913 \pm 1,181 \mathrm{r} /$ day). Table 3 shows the phenotypical alterations in our mouse lines in the presence and absence of RWs. Although body weights were different in both mouse lines, liver and muscle mass (M. quadriceps femoris) were 
Brenmoehl et al.: Dynamics of Fat Mass in DUhTP Mice Selected for Running

Performance - Fat Mobilization in a Walk

Table 3. Phenotypical traits of male mice long-term selected for high treadmill performance (DUhTP) and unselected controls (DUC) at an age of 70 days $^{\mathrm{a}}$

\begin{tabular}{|c|c|c|c|c|c|c|c|c|c|}
\hline & \multirow[t]{2}{*}{$\mathrm{n}$} & \multicolumn{3}{|l|}{ DUhTP } & \multicolumn{3}{|l|}{ DUC } & \multicolumn{2}{|l|}{$\mathrm{p}$ value } \\
\hline & & co & RW & $\mathrm{p}$ value & co & RW & $\mathrm{p}$ value & $\begin{array}{l}\text { DUhTP-co } \\
\text { vs. DUC-co }\end{array}$ & $\begin{array}{l}\text { DUhTP-RW } \\
\text { vs. DUC-RW }\end{array}$ \\
\hline Body mass, $g$ & 10 & $32.18 \pm 1.02$ & $31.25 \pm 0.87$ & 0.4925 & $35.18 \pm 0.67$ & $34.65 \pm 0.79$ & 0.6125 & 0.0191 & 0.0066 \\
\hline Lean body mass, $g$ & 10 & $20.89 \pm 0.59$ & $20.49 \pm 0.59$ & 0.6314 & $23.56 \pm 0.48$ & $23.45 \pm 0.54$ & 0.8693 & 0.0012 & 0.0007 \\
\hline Body length, cm & 10 & 10.0410 .12 & 9.9310 .11 & 0.4907 & $10.44 \pm 0.10$ & 10.4210 .09 & 0.8845 & 0.0135 & 0.0014 \\
\hline Musculus rectus femoris, $\mathrm{g}$ & 10 & $0.37 \pm 0.01$ & $0.38 \pm 0.01$ & 0.2969 & $0.39 \pm 0.01$ & $0.36 \pm 0.01$ & 0.0795 & 0.1378 & 0.1767 \\
\hline Liver mass, $\mathrm{g}$ & 10 & $1.81 \pm 0.07$ & $1.76 \pm 0.08$ & 0.6301 & $1.94 \pm 0.06$ & $1.82 \pm 0.06$ & 0.1513 & 0.1571 & 0.5192 \\
\hline Subcutaneous fat, g & 10 & $0.36 \pm 0.03$ & $0.22 \pm 0.03$ & 0.0012 & $0.20 \pm 0.01$ & $0.18 \pm 0.02$ & 0.4910 & 0.00003 & 0.2448 \\
\hline Epidymal fat, g & 10 & $0.33 \pm 0.03$ & $0.23 \pm 0.03$ & 0.0145 & $0.28 \pm 0.03$ & $0.20 \pm 0.02$ & 0.0183 & 0.2122 & 0.2718 \\
\hline Perinephric fat, g & 10 & $0.16 \pm 0.02$ & $0.08 \pm 0.01$ & 0.0020 & $0.09 \pm 0.01$ & $0.06 \pm 0.01$ & 0.0086 & 0.0035 & 0.2327 \\
\hline Brown fat, g & 10 & $0.09 \pm 0.01$ & $0.07 \pm 0.00$ & 0.0191 & $0.06 \pm 0.00$ & $0.06 \pm 0.00$ & 0.7512 & 0.0006 & 0.1189 \\
\hline Serum triglyceride, $\mathrm{mg} / \mathrm{ml}$ & $>7$ & $1.37 \pm 0.12$ & $0.96 \pm 0.08$ & 0.0079 & $1.15 \pm 0.12$ & $0.90 \pm 0.06$ & 0.0694 & 0.1999 & 0.5438 \\
\hline Serum cholesterol, $\mu \mathrm{g} / \mathrm{ml}$ & $>7$ & $1.97 \pm 0.09$ & $1.95 \pm 0.07$ & 0.8103 & $1.58 \pm 0.04$ & $1.63 \pm 0.07$ & 0.5841 & 0.0003 & 0.0039 \\
\hline Liver triglyceride, $\mu \mathrm{g} / \mathrm{mg}$ & $>7$ & $13.09 \pm 1.74$ & $6.43 \pm 0.33$ & 0.0049 & $6.25 \pm 0.32$ & $7.10 \pm 0.33$ & 0.1003 & 0.0042 & 0.1840 \\
\hline Liver cholesterol, $\mu \mathrm{g} / \mathrm{mg}$ & $>7$ & $3.72 \pm 0.24$ & $2.51 \pm 0.22$ & 0.0007 & $3.38 \pm 0.18$ & $3.61 \pm 0.20$ & 0.3937 & 0.2710 & 0.0007 \\
\hline
\end{tabular}

${ }^{\text {a }}$ Mice were kept in cages without (co) and with running wheels (RW). Physical activity was studied in RW for a period of 3 weeks in 7 - to 10 -week male mice of both lines. Total body lean mass was analyzed by dual energy X-ray absorptiometry. Vales are presented as mean \pm SE. Significances like indicated.

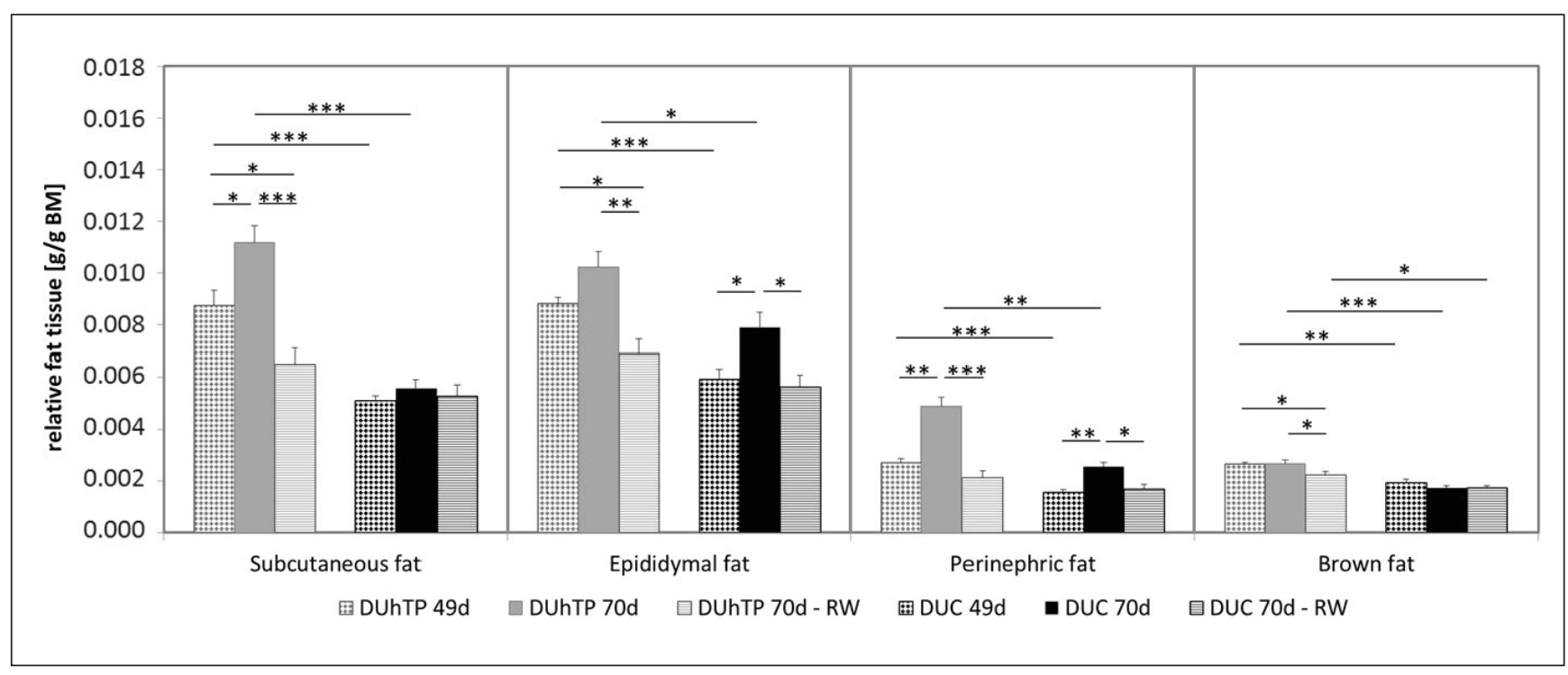

Fig. 1. Weights of subcutaneous, epididymal, perinephric, and brown fat depots normalized to body weight in 7- and 10-week-old male DUhTP (grew bars) and DUC mice (black bars) ( $\mathrm{n}=10)$. At an age of 7 weeks mice were kept in cages with (striped bars) or without RWs (filled bars) for a period of 3 weeks. Values are means $\pm \mathrm{SE} ;{ }^{*} \mathrm{p}<0.05,{ }^{* *} \mathrm{p}<0.005,{ }^{* * *} \mathrm{p}<0.0005$.

similar in all experimental groups. In DUhTP mice serum, cholesterol concentrations were 1.3 -fold $(\mathrm{p}<0.05)$ higher compared with controls. In the liver of DUhTP mice levels of TG were 2.1-fold higher than in DUC mice $(\mathrm{p}<0.01)$. In response to physical RW activity over a period of 3 weeks, exclusively DUhTP mice showed reduced concentrations of serum TG ( $p<$ $0.01)$ and lower levels of hepatic TG or hepatic cholesterol $(p<0.05)$. Liver TG and cholesterol were lower in DUhTP mice having access to RWs compared with DUC $(\mathrm{p}<0.001)$. At an age 
Fig. 2. a Effect of voluntary physical activity in RWs on protein expression of FAS (left panel) and LCAD (right panel) in subcutaneous fat tissue of 10-week-old male DUhTP and DUC mice. $\mathbf{b}$ The analysis was performed by Western immunoblotting. Protein expression was quantified by densitometry and normalized for the Coomassie blue signal. Data are presented as means and SE and are expressed relative to the expression level of control mice from line DUC (lower panel). Significant differences are indicated: *p $<0.05,{ }^{* *} \mathrm{p}<0.005$.

Brenmoehl et al.: Dynamics of Fat Mass in DUhTP Mice Selected for Running Performance - Fat Mobilization in a Walk

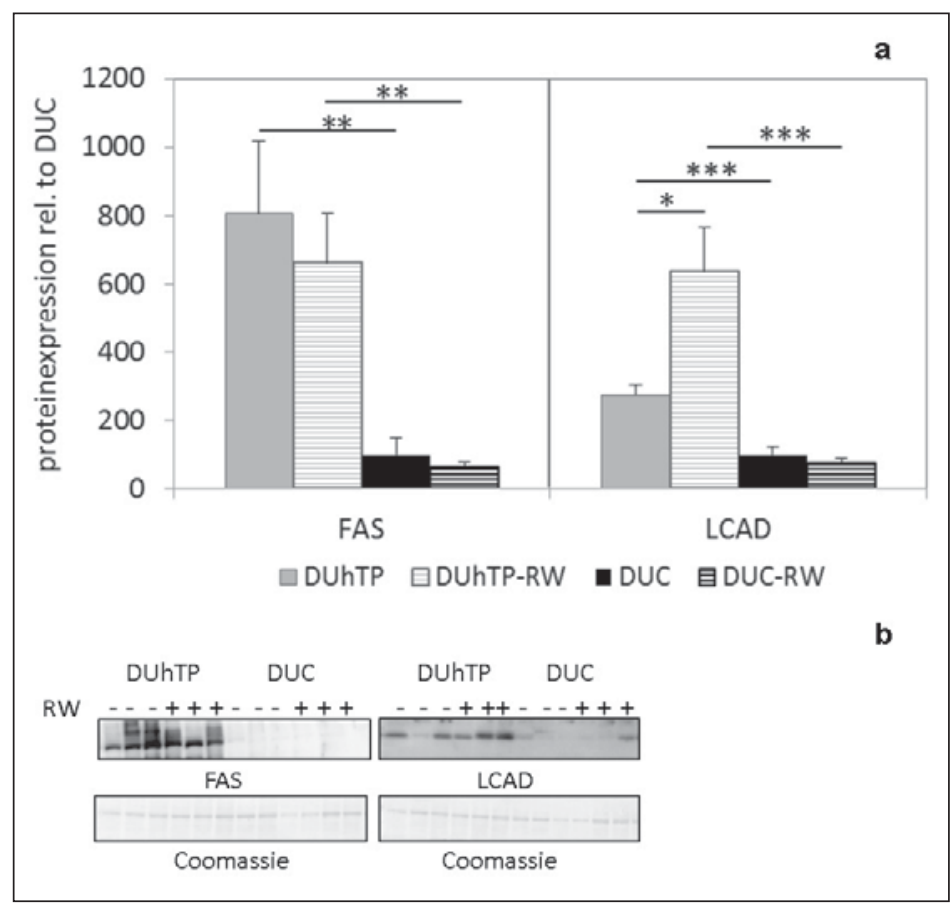

of 49 days, DUhTP mice were characterized by increased amounts of fat present in different fat depots (subcutaneous, epididymal, perinephric, and brown fat mass) compared with DUC mice. Highest differences of relative fat mass were observed for the subcutaneous and perinephric fat $(\approx 70 \%$ increase; fig. 1$)$. The marked increases in fat mass were even more pronounced at an age of 70 days in DUhTP mice (subcutaneous fat: $+100 \%$, perinephric fat +91\%; $\mathrm{p}<0.005$ ). Also for epididymal and brown fat tissues significant increases were present in 70-day-old DUhTP when compared to DUC mice ( $>30 \% ; \mathrm{p}<0.05$ ). Interestingly, voluntary physical activity in 70-day-old DUhTP mice significantly reduced absolute weights of all fat depots assessed compared with their sedentary 70-day-old littermates (table 2). If normalized for body mass, reductions were most obvious in perinephric fat $(-56 \%$; $<0.0005$; fig. 1) and subcutaneous fat $(-38 \%$; $<<0.0005)$ followed by epididymal and brown fat $(-32 \%$ and $-17 \%$, respectively; $\mathrm{p}<0.05)$. Voluntary physical activity also reduced fat mass in control DUC mice compared to sedentary littermates in the epididymal $(-28 \%)$ and perinephric fat depots $(-34 \%)$, while brown and subcutaneous fat depots remained unchanged. In subcutaneous and perinephric fat, we were able to observe age-related increases of fat accumulation between 49 days and 70 days of age $(+29 \%$ and $+80 \%$, respectively; $p<0.05)$. Moreover, physical activity reduced fat masses in subcutaneous, epididymal and brown fat $(-20 \%,-21 \%$ and $-15 \%$ respectively; $\mathrm{p}<0.05$ ) in 70 -day-old DUhTP mice not only when compared to sedentary littermates but also if compared to 49-day-old mice of the same line. Taken together, voluntary physical activity was able to eliminate the mouse line-specific accumulation of body fat in subcutaneous, epididymal, and perinephric tissues in DUhTP mice. Exclusively in subcutaneous fat, we were able to observe both a block of age-related fat increase as well as active degradation of existing body fat at an age of 49 days. We therefore focused on the control of energy metabolism in subcutaneous fat tissue in this subgroup.

\section{Expression of Fat-Producing and -Degrading Enzymes in Subcutaneous Fat}

At an age of 70 days subcutaneous fat tissue from DUhTP mice was characterized by massive expression of FAS in both experimental groups (fig. 2, left panel). In sedentary mice, 
Fig. 3. Effect of voluntary physical activity in RWs on relative mRNA (a) and protein (b) levels of PGC$1 \mathrm{a}$ in subcutaneous adipose tissue from DUhTP and DUC. Analysis of mRNA expression was performed by qPCR and normalized to expression of HKG Rplp2. c Protein analysis was performed by Western immunoblotting. Protein expression was quantified by densitometry and normalized for the Coomassie blue signal. All data are expressed relative to the expression level of sedentary unselected controls (DUC) with no access to RW (lower panel). Values are means $\pm \mathrm{SE} ;{ }^{*} \mathrm{p}<0.05, * * \mathrm{p}$ $<0.005,{ }^{* * *} \mathrm{p}<0.0005$.
Brenmoehl et al.: Dynamics of Fat Mass in DUhTP Mice Selected for Running Performance - Fat Mobilization in a Walk

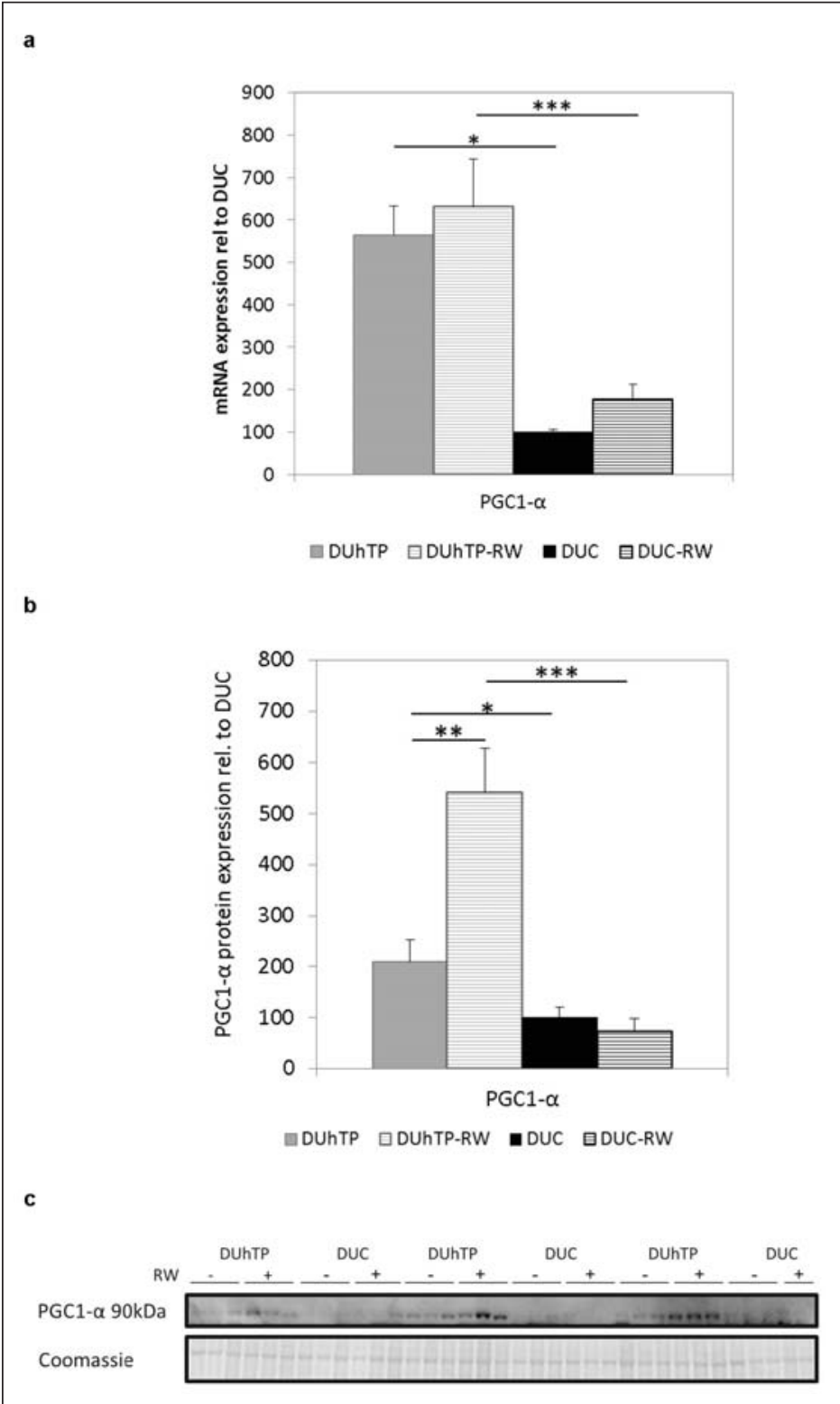

FAS expression in DUhTP animals was about eightfold $(\mathrm{p}<0.005)$ higher compared to DUC mice, indicating higher lipid synthesis in subcutaneous fat. In the presence of RWs, the high level of FAS expression was maintained in DUhTP mice, and voluntary physical activity did not have any effect on FAS expression in both mouse lines. Furthermore, expression of the lipiddegrading enzyme LCAD was elevated in sedentary DUhTP versus DUC mice (threefold; $p<$ 0.0005). Physical activity over a period of 3 weeks further increased LCAD expression (2.3fold; $p<0.05$ ) in subcutaneous fat tissue of DUhTP, but not of DUC mice (fig. 2, right panel).

\section{Mitochondrial Biogenesis in Subcutaneous Fat}

The more than twofold increase of LCAD expression in subcutaneous fat in response to physical activity led us to investigate PGC1- $\alpha$-mediated mitochondrial biogenesis in our system. PGC1- $\alpha$ mRNA expression was significantly increased in DUhTP mice (5.6-fold; $p<0.0005$ ) compared to controls. Physical activity as a trend increased mRNA expression of PGC1- $\alpha$ in 
Brenmoehl et al.: Dynamics of Fat Mass in DUhTP Mice Selected for Running

Performance - Fat Mobilization in a Walk

subcutaneous fat of both mouse lines $(12 \%$ or $77 \%)$. The transcription data was confirmed via immunoblot by higher expression of the PGC1- $\alpha$ isoform 1 in sedentary DUhTP mice (2.1-fold; $\mathrm{p}<0.05$; fig. $3 \mathrm{~b}$ ). Interestingly, physical activity significantly increased protein levels of PGC1- $\alpha$ $(+157 \%$; $p<0.0005)$ in DUhTP, but not in DUC mice. To further address PGC1- $\alpha$-mediated induction of mitochondrial biogenesis, we investigated protein expression of Tfam, mitochondria-encoded subunit ND-1 of respiratory chain complex I, NDUFA9 as well as mitochondrial SIRT3 (fig. 4). In total subcutaneous fat tissue extracts, two isoforms of Tfam (fig. 4a) were detected (mitochondrial Tfam: $29 \mathrm{kDa}$; nuclear Tfam: $25 \mathrm{kDa}$; Uni-Prot P40630). DUhTP mice as a trend showed increased levels of mitochondrial Tfam $(+41 \%)$, whereas protein levels of nuclear Tfam were similar in both mouse lines. Mitochondrial and nuclear Tfam was significantly elevated in response to physical activity in DUhTP mice only $(+40 \%$ and $+75 \%$, respectively; $\mathrm{p}<0.05$ ). Tfam-related expression of ND-1 in mitochondria (fig. 4a) was also significantly increased in DUhTP compared with control mice $(+76 \%$; $<<0.05)$. In the presence of RWs, protein expression of ND-1 was elevated as a trend by 31\% (DUhTP) and 25\% (DUC). In sedentary DUhTP mice, protein levels of complex I subunit NDUFA-9 and SIRT3 (fig. 4b) were increased if compared to DUC mice. In mitochondria, physical activity significantly increased protein levels exclusively in DUhTP mice (NDUFA9: 1.5-fold, $\mathrm{p}<0.05$; SIRT3: about 2.3-fold, $\mathrm{p}$ $<0.05)$. Furthermore, in subcutaneous fat of DUhTP mice, a 16-fold increase of mtDNA content was observed when compared to DUC mice ( $p<0.0001$; fig. 4c). Physical activity over a period of 3 weeks further increased mtDNA content in DUhTP and DUC mice (7.8-fold and 7.7-fold, respectively; $p<0.05$ ). Interestingly, in contrast to subcutaneous fat of controls, that of DUhTP mice has less clustering of mitochondria around the nucleus and mitochondrial fragmentation (fig. 4c). In response to RW activity more mitochondrial mass is detectable in subcutaneous fat of both genotypes. In controls, formation of elongated mitochondria appears in combination with mitochondrial clustering in the cell because of alterations in mitochondrial fission. Only in DUhTP mice a homogenous mitochondrial network structure was found across the cell.

\section{Discussion}

We have previously described a novel genetic mouse model (DUhTP) established by long-term selection for high treadmill performance that is characterized by genetically fixed elevated running capacity, increased hepatic lipogenesis, and peripheral obesity compared to control mice (DUC) [8]. We now examined energy metabolism and mitochondrial biogenesis in our mouse model with and without voluntary physical activity. As expected and observed in other studies in mice $[28,29]$ or humans [24], voluntary physical activity efficiently blocked the accumulation of body fat in peripheral fat depots from both genetic groups if compared to sedentary controls. In addition, prominent reductions of existing fat mass were found in perinephric and subcutaneous fat tissues exclusively in DUhTP mice in response to mild physical exercise over a period of 3 weeks. This was surprising, because the extent of voluntary physical activity in the RWs is similar in DUhTP mice and controls as described before [8]. In particular, physical activity is thought to exert beneficial effects on health through induction of lipolytic activity within adipose tissues [30]. In Osborne-Mendel rats, Zachwieja and colleagues [31] detected reduced fat masses in inguinal, epididymal, retroperitoneal, and perirenal fat pads in response to exercise for 7 weeks. Vieira et al. [32] could demonstrate a negative effect of physical activity ( $40 \mathrm{~min} /$ day over 12 weeks) on epididymal fat mass and hepatic TG levels. In DUhTP mice, we detected reduced hepatic cholesterol and TG content and serum TG levels by voluntary physical activity. While other studies have applied specific exercise training protocols over a period of 6-12 weeks [32], in our experiment merely 3 weeks of voluntary physical activity were sufficient to reduce hepatic TG and cholesterol in 
Brenmoehl et al.: Dynamics of Fat Mass in DUhTP Mice Selected for Running Performance - Fat Mobilization in a Walk

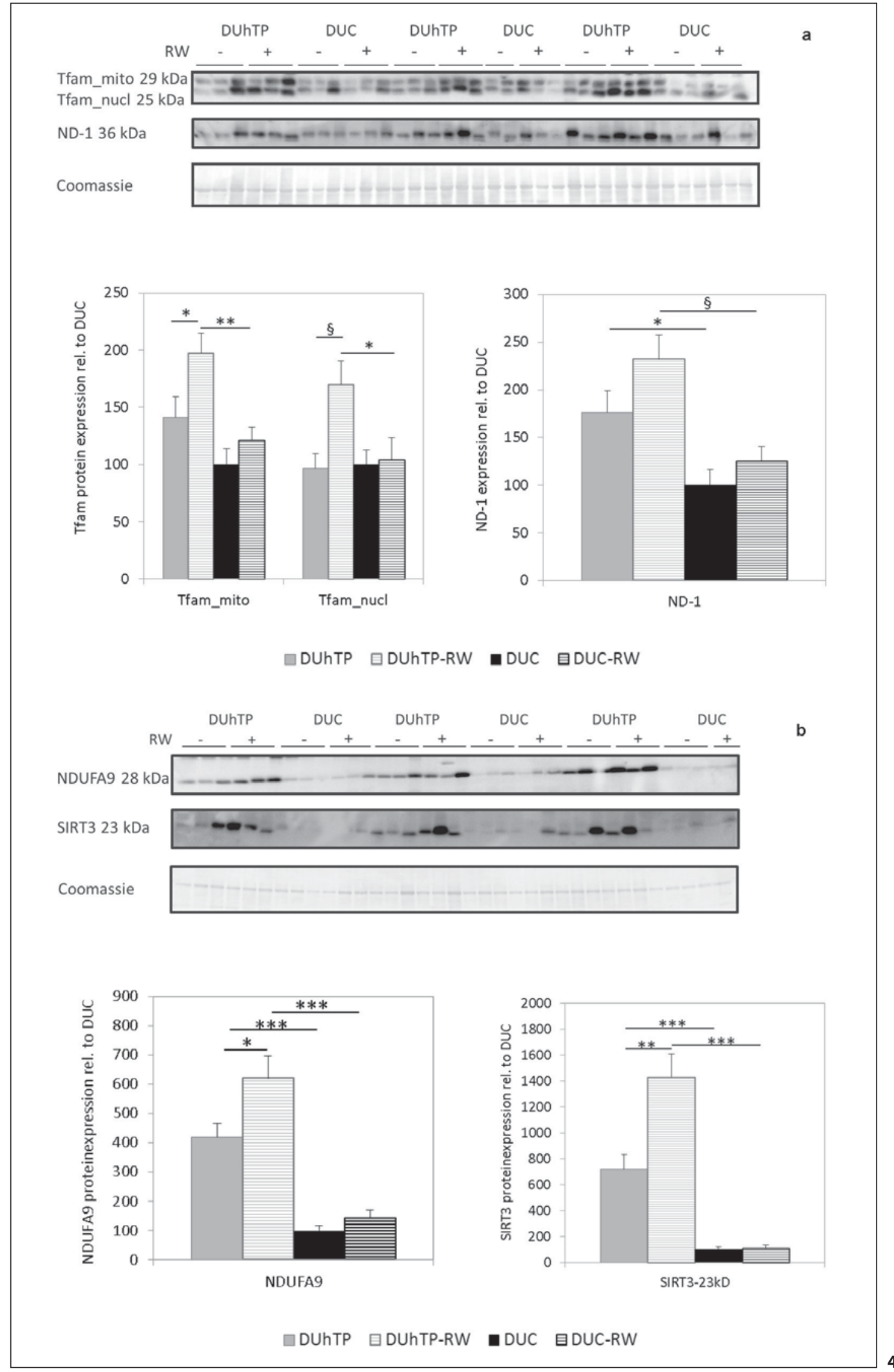

(For legend see next page.) 


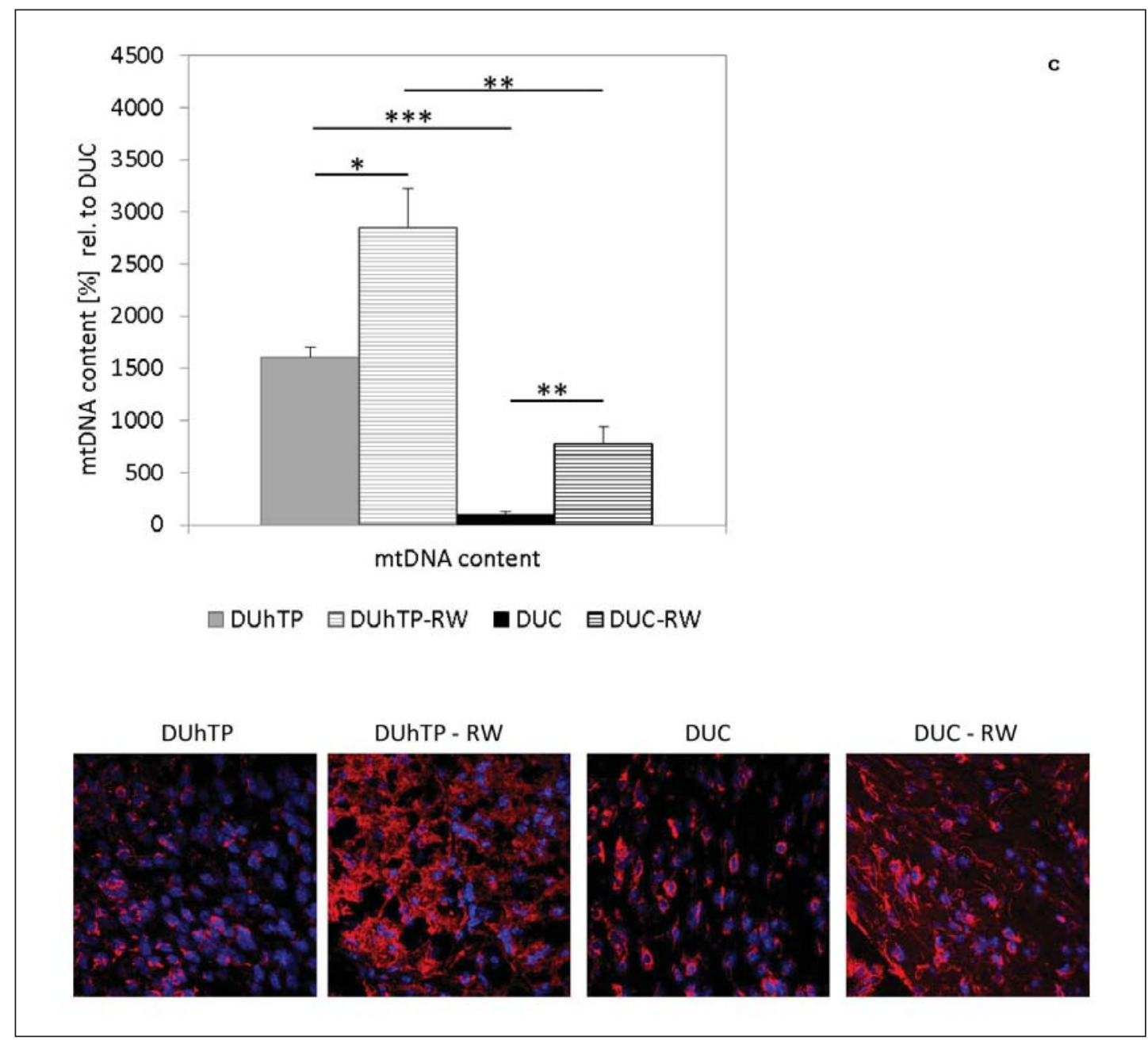

Fig. 4. Effect of voluntary physical activity in RWs on the expression of mitochondrial transcription factor A (Tfam) and mitochondria-encoded complex I subunit ND-1 (a) and mitochondrial proteins complex I subunit NDUFA9 and SIRT3 (b) as well as mtDNA content and mitochondrial biogenesis (c) in subcutaneous fat tissue of 10-week male DUhTP and DUC. Mitochondrial staining was achieved using Mitotracker Deep Red in fixed tissues as described in 'Material and Methods'. The analysis was performed by Western immunoblotting. Protein expression was quantified by densitometry and normalized for the Coomassie blue signal. Data are expressed relative to the expression level of the sedentary unselected controls (DUC). Vales are presented as mean \pm SE. Significances are indicated; ${ }^{*} \mathrm{p}<0.05,{ }^{\S} \mathrm{p}<0.01,{ }^{* *} \mathrm{p}<0.005,{ }^{* * *} \mathrm{p}<0.0005$.

DUhTP mice. In addition, the previous study was focused on the analysis of endpoints in divergently selected rats, whereas we have studied control of fat metabolism in different age groups in the presence or absence of RWs in DUhTP mice.

Expression of FAS and LCAD was higher in subcutaneous fat from DUhTP mice than in that of control mice, and expression of LCAD was further increased by physical activity in DUhTP mice. Also in human muscles, gene expression of LCAD increased after $8 \mathrm{~h}$ in response to 30 min single bout of exercise [25], and in muscle of rats, already in 1971, Mole and colleagues [12] could detect a twofold increased oxidation of long-chain fatty acids like oleate or linoleate in response to exercise on a treadmill over 12 weeks. In our model increased expression of LCAD in response to physical activity might correlate with increased $\beta$-oxidation 
Brenmoehl et al.: Dynamics of Fat Mass in DUhTP Mice Selected for Running Performance - Fat Mobilization in a Walk

in mitochondria on the one hand and a reduction of fat mass on the other. Thus we may assume a higher mitochondrial mass established by increased mitochondrial biogenesis in DUhTP in response to RW activity. In fact very recent work provided evidence for exerciseinduced mitochondrial biogenesis in subcutaneous fat in mice [16]. Exercise-induced mitochondrial biogenesis in adipose tissues so far has been considered only by a comparably small number of studies. In muscle or adipose tissues, higher concentrations of PGC1- $\alpha$, a potent effector of mitochondrial biogenesis, were found in response to exercise training $[15,16,22]$. In murine preadipocytes, PGC1- $\alpha$ and PPAR $\alpha$ induce gene expression of LCAD involved in mitochondrial fatty acid oxidation [33]. PGC1- $\alpha$ stimulates mitochondrial biogenesis in terms of higher mtDNA content and modulates regulators of mitochondrial replication and transcription in myotubes [22]. PGC1- $\alpha$ controls mitochondria- and nucleus-encoded genes involved in mitochondrial respiration and oxidative phosphorylation in cardiac myocytes and muscle cells [22, 34]. Most interestingly DUhTP mice synthesize severalfold higher levels of PGC1- $\alpha$ in their subcutaneous fat, if compared to controls. Expression of PGC1- $\alpha$ was further increased by physical activity only in DUhTP mice. Surprisingly, in contrast to muscle $[20,35]$ or heart [35] only one form of PGC1- $\alpha(90 \mathrm{kDa})$ was observed in subcutaneous fat corresponding to PGC1- $\alpha$ isoform 1.

Transcription of mitochondrial-encoded genes is mediated by Tfam in HeLa cells and Tfam expression again is induced in nucleus by PGC1- $\alpha$ via NRF-1, which identifies the latter two as bi-genomic coordinators of respiratory subunit expression [36]. Tfam is imported into mitochondrial subcompartments inducing transcription of $13 \mathrm{mtDNA}$-encoded protein subunits, which are essential components of the mitochondrial electron transport chain [37]. In sedentary DUhTP and control mice, mitochondrial or nuclear Tfam protein levels were on a comparable level. Nevertheless, voluntary physical activity significantly increased levels of both mitochondrial and nuclear Tfam in DUhTP, but not in DUC mice. In epididymal and retroperitoneal fat patches of male Wistar rats [15] or in subcutaneous fat of mice [16], an increase in mitochondrial biogenesis accompanied by higher PGC1- $\alpha$ and Tfam mRNA levels was found after 4 or 6 weeks of exercise swim training. Directly after an acute 2-hour bout of swimming only PGC1- $\alpha$ gene expression was increased in WAT, whereas mRNA concentration of Tfam was unchanged [15]. PGC1- $\alpha$ has been shown to induce expression of proteins encoded by the mitochondria such as ND-1 to ND-4 and ND-6 as markers of mitochondrial biogenesis and compounds of the respiratory chain in human and murine muscles, in the hippocampus and Corpus striatum regions of rat brain, and in rat kidneys $[24,25]$. Both in the presence and absence of RWs, protein expression of ND-1 was higher in DUhTP mice than in controls. In response to physical activity, ND-1 expression increased slightly in both genotypes but the increase did not reach statistical significance.

In addition, nucleus-encoded mitochondrial proteins SIRT3 and NDUFA9 were examined to support the hypothesis of elevated mitochondrial biogenesis in DUhTP mice. Both proteins are expressed in a PGC1- $\alpha$-dependent and Tfam-independent fashion [38]. In fact, in subcutaneous fat of sedentary DUhTP mice, higher protein levels of SIRT3 and NDUFA9 were detected when compared to controls. Again only in DUhTP mice voluntary activity further increased expression of SIRT3 and NDUFA9. It is known that PGC1- $\alpha$ induces gene expression of SIRT3 [38] while inhibition of SIRT3 expression blocked PGC1- $\alpha$-mediated mitochondrial biogenesis [38], potentially since SIRT3 is required for the activation of NDUFA9 by deacetylation [39]. In addition, an increased content of mitochondrial DNA was found in DUhTP mice. In response to RW activity, mtDNA levels were elevated in DUhTP and DUC mice. In subcutaneous fat of DUhTP mice, physical activity coincides with the presence of a homogenous mitochondrial network distributed across the cell, maintaining cellular function and respiratory capacity. These dynamic networks continuously undergo fusion and fission events that allow damaged mitochondria to recover its activity and maintain metabolic functions, whereas 
Brenmoehl et al.: Dynamics of Fat Mass in DUhTP Mice Selected for Running

Performance - Fat Mobilization in a Walk

dysfunctional mitochondria get removed from the network by autophagy. The correct balance between fusion and fission of mitochondria is required for normal tubular morphology. An imbalance of this process results in fragmentation, elongation, or clustering of mitochondria [40] and a lack of mitophagy [41]. Future studies are designed also to address $\mathrm{CO}_{2}$ consumption and body temperature in our mouse model.

\section{Conclusions}

To summarize, we provide direct evidence that subcutaneous fat from DUhTP mice is characterized by elevated expression of LCAD, PGC1- $\alpha$, ND-1, NDUFA9, SIRT3 and increased mtDNA, which per se are not sufficient to induce subcutaneous fat mobilization. By contrast, in DUhTP mice even voluntary physical activity on RWs is sufficient to induce efficient lipolytic activity, which is reflected by a substantial loss of fat mass in different depots. Moderate physical activity further increased levels of LCAD, PGC1- $\alpha$, Tfam, NDUFA9, and SIRT3 as well as mitochondrial biogenesis in subcutaneous fat from DUhTP mice, but not from controls. We hypothesize that during long-term selection DUhTP mice have acquired a set of energy-metabolic adaptations in order to fuel the high energy demands during physical activity. These adaptations confer the capacities to burn down existing fat mass even during comparably mild voluntary physical activity.

\section{Acknowledgements}

The authors want to thank Luong Chau, Sabine Hinrichs, Sabine Geist, Benita Lucht, Karin Ullerich, Sonja Alm and Magdalene Bülau for excellent technical assistance and Gordon Lynch for help with the manuscript. This study was supported by the Deutsche Forschungsgemeinschaft (DFG HO 2003/6-1).

\section{Disclosure Statement}

The authors declare no conflict of interest.

\section{References}

1 Feng B, Zhang T, Xu H: Human adipose dynamics and metabolic health. Ann N Y Acad Sci 2013;1281:160-177.

2 Frayn KN: Fat as a fuel: emerging understanding of the adipose tissue-skeletal muscle axis. Acta Physiol (Oxf) 2010;199:509-518.

3 Melzer K: Carbohydrate and fat utilization during rest and physical activity. e-SPEN 2011;6:e45-e52.

4 Romijn JA, Coyle EF, Sidossis LS, et al: Regulation of endogenous fat and carbohydrate metabolism in relation to exercise intensity and duration. Am J Physiol 1993;265:E380-E391.

5 Goto K, Ishii N, Sugihara S, Yoshioka T, Takamatsu K: Effects of resistance exercise on lipolysis during subsequent submaximal exercise. Med Sci Sports Exerc 2007;39:308-315.

6 Kang J, Rashti SL, Tranchina CP, Ratamess NA, Faigenbaum AD, Hoffman JR: Effect of preceding resistance exercise on metabolism during subsequent aerobic session. Eur J Appl Physiol 2009;107:43-50.

7 Wang S, Moustaid-Moussa N, Chen L, et al: Novel insights of dietary polyphenols and obesity. J Nutr Biochem 2014;25:1-18.

8 Brenmoehl J, Walz C, Renne U, et al: Metabolic adaptations in the liver of born long-distance running mice. Med Sci Sports Exerc 2013;45:841-850.

9 Wright DC: Exercise- and resveratrol-mediated alterations in adipose tissue metabolism. Appl Physiol Nutr Metab 2014;39:109-116.

10 Holloszy JO: Biochemical adaptations in muscle. Effects of exercise on mitochondrial oxygen uptake and respiratory enzyme activity in skeletal muscle. J Biol Chem 1967;242:2278-2282.

11 Holloszy JO, Oscai LB, Don IJ, Mole PA. Mitochondrial citric acid cycle and related enzymes: adaptive response to exercise. Biochem Biophys Res Commun. 1970;40(6):1368-1373. 
Brenmoehl et al.: Dynamics of Fat Mass in DUhTP Mice Selected for Running

Performance - Fat Mobilization in a Walk

12 Mole PA, Oscai LB, Holloszy JO: Adaptation of muscle to exercise. Increase in levels of palmityl Coa synthetase, carnitine palmityltransferase, and palmityl Coa dehydrogenase, and in the capacity to oxidize fatty acids. J Clin Invest 1971;50:2323-2330.

13 Horowitz JF, Leone TC, Feng W, Kelly DP, Klein S: Effect of endurance training on lipid metabolism in women: a potential role for PPARalpha in the metabolic response to training. Am J Physiol Endocrinol Metab 2000; 279:E348-E355.

14 Jeppesen J, Kiens B: Regulation and limitations to fatty acid oxidation during exercise. J Physiol 2012;590: 1059-1068.

15 Sutherland LN, Bomhof MR, Capozzi LC, Basaraba SA, Wright DC: Exercise and adrenaline increase PGC1 alpha\} mRNA expression in rat adipose tissue. J Physiol 2009;587:1607-1617.

16 Trevellin E, Scorzeto M, Olivieri M, et al: Exercise training induces mitochondrial biogenesis and glucose uptake in subcutaneous adipose tissue through eNOS-dependent mechanisms. Diabetes. 2014;63:2800-2811.

17 Hashimoto T, Sato K, Iemitsu M: Exercise-inducible factors to activate lipolysis in adipocytes. J Appl Physiol 2013;115:260-267.

18 Summermatter S, Handschin C: PGC-1alpha and exercise in the control of body weight. Int J Obes (Lond) 2012; $36: 1428-1435$

19 Summermatter S, Troxler H, Santos G, Handschin C: Coordinated balancing of muscle oxidative metabolism through PGC-1alpha increases metabolic flexibility and preserves insulin sensitivity. Biochem Biophys Res Commun 2011:408:180-185.

20 Brenmoehl J, Albrecht E, Komolka K, et al: Irisin is elevated in skeletal muscle and serum of mice immediately after acute exercise. Int J Biol Sci 2014;10:338-349.

21 Evans MJ, Scarpulla RC: NRF-1:a trans-activator of nuclear-encoded respiratory genes in animal cells. Genes Dev 1990;4:1023-1034.

22 Wu Z, Puigserver P, Andersson U, et al: Mechanisms controlling mitochondrial biogenesis and respiration through the thermogenic coactivator PGC-1. Cell 1999;98:115-124.

23 Ryan MT, Hoogenraad NJ: Mitochondrial-nuclear communications. Annu Rev Biochem 2007;76:701-722.

24 Short KR, Vittone JL, Bigelow ML, et al: Impact of aerobic exercise training on age-related changes in insulin sensitivity and muscle oxidative capacity. Diabetes 2003;52:1888-1896.

25 Fluck M: Functional, structural and molecular plasticity of mammalian skeletal muscle in response to exercise stimuli. J Exp Biol 2006;209:2239-2248.

26 Safdar A, Little JP, Stokl AJ, Hettinga BP, Akhtar M, Tarnopolsky MA: Exercise increases mitochondrial PGC1alpha content and promotes nuclear-mitochondrial cross-talk to coordinate mitochondrial biogenesis. J Biol Chem 2011;286:10605-10617.

27 Hofmeister-Brix A, Kollmann K, Langer S, Schultz J, Lenzen S, Baltrusch S: Identification of the ubiquitin-like domain of midnolin as a new glucokinase interaction partner. J Biol Chem 2013;288:35824-35839.

28 Colman RJ, Nam G, Huchthausen L, Mulligan JD, Saupe KW: Energy restriction-induced changes in body composition are age specific in mice. J Nutr 2007;137:2247-2251.

29 Leamy LJ, Kelly SA, Hua K, Pomp D: Exercise and diet affect quantitative trait loci for body weight and composition traits in an advanced intercross population of mice. Physiol Genomics 2012;44:1141-1153.

30 Stephenson EJ, Lessard SJ, Rivas DA, et al: Exercise training enhances white adipose tissue metabolism in rats selectively bred for low- or high-endurance running capacity. Am J Physiol Endocrinol Metab 2013;305:E429E438.

31 Zachwieja JJ, Hendry SL, Smith SR, Harris RB: Voluntary wheel running decreases adipose tissue mass and expression of leptin mRNA in Osborne-Mendel rats. Diabetes 1997;46:1159-1166.

32 Vieira VJ, Valentine RJ, Wilund KR, Antao N, Baynard T, Woods JA: Effects of exercise and low-fat diet on adipose tissue inflammation and metabolic complications in obese mice. Am J Physiol Endocrinol Metab 2009; 296:E1164-E1171.

33 Vega RB, Huss JM, Kelly DP: The coactivator PGC-1 cooperates with peroxisome proliferator-activated receptor alpha in transcriptional control of nuclear genes encoding mitochondrial fatty acid oxidation enzymes. Mol Cell Biol 2000;20:1868-1876.

34 Lehman JJ, Barger PM, Kovacs A, Saffitz JE, Medeiros DM, Kelly DP: Peroxisome proliferator-activated receptor gamma coactivator-1 promotes cardiac mitochondrial biogenesis. J Clin Invest 2000;106:847-856.

35 Ruas JL, White JP, Rao RR, et al: A PGC-1alpha isoform induced by resistance training regulates skeletal muscle hypertrophy. Cell 2012;151:1319-1331.

36 Virbasius JV, Scarpulla RC: Activation of the human mitochondrial transcription factor A gene by nuclear respiratory factors: a potential regulatory link between nuclear and mitochondrial gene expression in organelle biogenesis. Proc Natl Acad Sci U S A 1994;91:1309-1313.

37 Menzies KJ, Hood DA: The role of SirT1 in muscle mitochondrial turnover. Mitochondrion 2012;12:5-13.

38 Kong X, Wang R, Xue Y, et al: Sirtuin 3, a new target of PGC-1alpha, plays an important role in the suppression of ROS and mitochondrial biogenesis. PLoS One 2010;5:e11707.

39 Ahn BH, Kim HS, Song S, et al: A role for the mitochondrial deacetylase Sirt3 in regulating energy homeostasis. Proc Natl Acad Sci U S A. 2008;105:14447-14452.

40 Huang $\mathrm{P}, \mathrm{Yu}$ T, Yoon Y: Mitochondrial clustering induced by overexpression of the mitochondrial fusion protein Mfn2 causes mitochondrial dysfunction and cell death. Eur J Cell Biol 2007;86:289-302.

41 Mouli PK, Twig G, Shirihai OS: Frequency and selectivity of mitochondrial fusion are key to its quality maintenance function. Biophys J 2009;96:3509-3518. 